CARTA AL EDITOR

\section{CONSIDERACIONES METODOLÓGICAS SOBRE EL ARTÍCULO «USO DE TERAPIAS DE MEDICINA ALTERNATIVA Y COMPLEMENTARIA EN LA PROVINCIA DE CORONEL PORTILLO, UCAYALI, PERÚ»}

\section{METHODOLOGICAL CONSIDERATIONS ON THE ARTICLE «USE OF COMPLEMENTARY AND ALTER- NATIVE MEDICINE THERAPIES IN THE CORONEL PORTILLO PROVINCE, UCAYALI, PERU»}

\author{
Rodrigo Vargas-Fernández (D1,a \\ 1 Universidad Científica del Sur, Lima, Perú \\ a Médico cirujano
}

Sr. Editor: He leído con detenimiento el artículo de Santiváñez-Acosta et al. ${ }^{(1)}$ publicado en el número 3 del 2020 de la revista, el cual es un estudio de tipo transversal analítico realizado en tres distritos (Callería, Manantay y Yarinacocha) del departamento de Ucayali, donde consideraron un total de 67156 viviendas distribuidas en los tres distritos de estudio para obtener una muestra, mediante un muestreo aleatorio simple. Los autores estimaron un tamaño de muestra de 840 encuestados (395 encuestados en Callería, 252 en Yarinacocha y 193 en Manantay), cabe destacar que las encuestas fueron realizadas sólo a un miembro de la familia por cada vivienda. Sin embargo, al momento de replicar el cálculo del tamaño muestral con los parámetros establecidos por los autores y utilizando el programa Epidat versión 3.1, no se obtiene el mismo tamaño muestral.

Para replicar el cálculo del tamaño muestral, se consideró al total de viviendas seleccionadas por los autores (67 156), una proporción esperada del $50 \%$ de uso de medicina alternativa y complementaria (MAC), un nivel de confianza del $95 \%$ y una precisión del $5 \%$, con lo que se obtuvo un tamaño de muestra final de 382 encuestados (Tabla 1). A pesar de contar con una tasa de no respuesta del 10\%, la muestra replicada fue muy disímil de los 840 encuestados

\footnotetext{
Citar como: Vargas-Fernández R. Consideraciones metodológicas sobre el artículo «Uso de terapias de medicina alternativa y complementaria en la provincia de Coronel Portillo, Ucayali, Perú». 2021;38(1):188-9. doi: https:// doi.org/10.17843/rpmesp.2021.381.6587.
}

Correspondencia: Rodrigo Vargas-Fernández; Carretera Panamericana Sur km. 19, Villa El Salvador. Lima, Perú; 100006379@ucientifica.edu.pe

Recibido: 19/10/2020 Aprobado: 28/10/2020 En línea: 05/02/2021
Tabla 1. Cálculo del tamaño de muestra

\begin{tabular}{lc}
\hline Parámetros & Resultados \\
\hline Tamaño de la población & 67156 \\
Proporción esperada & $50 \%$ \\
Nivel de confianza & $95 \%$ \\
Precisión & $5 \%$ \\
Tamaño de muestra & 382 \\
\hline
\end{tabular}

estimados por los autores. Tomando en consideración esta diferencia se podría precisar que, existe un error en el cálculo del tamaño muestral que podría afectar la validez y precisión de los estimados que, llevaría a cuestionar la confiabilidad de los resultados del estudio.

Santiváñez-Acosta et al. ${ }^{(1)}$ estimaron más del doble del tamaño muestral replicado en la presente carta, este error tiene implicancias en la inferencia estadística que se requiere para poder extrapolar los resultados a la población objetivo; además, tiene repercusiones en la potencia estadística, porque aumentaría la probabilidad de detectar diferencias significativas entre el uso de la MAC y las variables independientes ${ }^{(2)}$, y llevaría a cometer un error de tipo 1 (se rechaza la hipótesis nula cuando es verdadera) ${ }^{(3)}$, lo que podría generar un error de interpretación de los factores asociados ${ }^{(4)}$. Por estas razones, se debe considerar calcular un tamaño muestral adecuado en un estudio transversal y la prevalencia en la población objetivo; para ello, se necesitan seleccionar parámetros requeridos en la siguiente fórmula ${ }^{(5)}$

$$
n=\frac{Z^{2}[P(1-P)]}{d^{2}}
$$

Donde $n$ es el tamaño de la muestra, $Z$ es el valor de la distribución para tener un intervalo de confianza del 95\%, $P$ es la prevalencia esperada estimada a partir de estudios previos u obtenida de un estudio piloto y $d$ es la precisión del intervalo de confianza que usualmente es $5 \%$, y que depende de la prevalencia esperada. Cabe resaltar que, para el cálculo del tamaño de muestra, los autores asumieron una prevalencia esperada del 50\% como un valor estándar para obtener el valor máximo del tamaño muestral, el cual no fue tomado de la literatura o de un estudio piloto, como diversos autores lo recomiendan ${ }^{(5)}$.

En conclusión, todo estudio necesita tener un tamaño de muestra suficiente o mínimo a fin de hacer conclusiones veraces y confiables de los resultados.

Contribuciones de autoría: El autor buscó información, redactó y revisó críticamente el manuscrito, y aprobó la versión final.

Financiamiento: Autofinanciado.

Conflictos de interés: El autor declara no tener conflictos de interés. 


\section{REFERENCIAS BIBLIOGRÁFICAS}

1. Santiváñez-Acosta R, Valenzuela-Oré F, Angulo-Bazán Y. Uso de terapias de medicina alternativa y complementaria en la provincia de Coronel Portillo, Ucayali, Perú. Rev Peru Med Exp Salud Publica. 2020;37(3):510-5. doi: 10.17843/rpmesp.2020.373.4939.

2. Sedgwick P. Pitfalls of statistical hypothesis testing: type I and type II errors. BMJ. 2014;349:g4287. doi: 10.1136/bmj.g4287.
3. Fosgate GT. Practical sample size calculations for surveillance and diagnostic investigations. J Vet Diagnostic Investig. 2009;21(1):3-14. doi: 10.1177/104063870902100102.

4. Dahiru T, Aliyu A, Kene TS. Statistics in medical research: Misuse of sampling and sample size determination. Ann Afr Med. 2006;5(3):158.

5. Pourhoseingholi MA, Vahedi M, Rahimzadeh M. Sample size calculation in medical studies. Gastroenterol Hepatol from Bed to Bench. 2013;6(1):14-7. doi: 10.22037/ghfbb.v6i1.332. 